\title{
HIS-RECT Nonlinear Compandique Technique for MC-CDMA MIMO Transmission
}

\author{
Varus Mbembo Loundou, Jie Yang, Nouhoum S. A. Galeb Yari, and Dong Doan Van
}

\begin{abstract}
Multi Carrier-Code Division Multiple Access (MC-CDMA) in multicarrier modulation system is considered as the new reliable candidate to offer high data transmission in mobile environment; but the presence of High Peak to Average Power Ratio (PAPR) is considered as a critical major drawback since it is present in OFDM-MC CDMA scheme. it constitutes an obstacle in its use and its presence in non-linear region of the power amplifier, causing signal distortion by producing an inefficient amplification power in the transmission system. In this paper, after review and making comparison with main current performed Nonlinear Compandique techniques in the integral context of OFDM and MC-CDMA PAPR reduction techniques, we propose a novel nonlinear companding transform (NLT) technique based on the combination of two recent NCT based techniques named hyperbolic sine function and the revised exponential companding transform called (HIS-RECT). By introducing more adjustability in companding function and an effective trade-off between the PAPR and bit rate error (BER) performance can be offered to get stable high power amplified (HPA) and the non affectation of cost in the system. Results shows that this novel technique approach outperform existing NTC techniques for MC-CDMA high data transmission. The 11dB PAPR reduction performance result is obtained with Bit Error Rate (BER) improved and better Power Spectral density/bandwidth.
\end{abstract}

Index Terms-Telecommunications, up-link, HPA, MC-CDMA, MIMO transmission.

\section{INTRODUCTION}

Multicarrier Code Division Multiple Access is a technique which results of combination of orthogonal frequency division multiplexing (OFDM) and code division multiple access (CDMA), taking advantage of both the techniques: OFDM multi-carrier transmission counteracts frequency selective fading channels and reduces signal processing complexity by enabling equalization in the frequency domain, whereas CDMA spread spectrum technique allows the multiple access using an assigned spreading code for each user, thus minimizing the access interference (MAI) (K. Fazel, 2003; Hanzo \& Keller, 2006). The advantages of multi-carrier modulation on one hand and the flexibility offered by the spread spectrum technique on the other hand let MC-CDMA be a candidate technique for next generation mobile wireless systems and especially in high data transmission process where spectral efficiency and flexibility are considered as the most important criteria for the choice of the air interface. And this technique offer also another advantage due to the recent advances in digital signal

Manuscript received May 12, 2016; revised August 10, 2016.

The authors are with Wuhan University of Technology, Information Engineering Department, China (e-mail: loundouvarus@yahoo.fr, 466802324@qq.com, yasmigab@yahoo.fr, dongdoan2211@yahoo.com). processing technology.

However, the main drawback of the use of Multicarrier Code Division Multiple Access (MC-CDMA) is the high presence of high Peak-to-Average Power Ratio (PAPR) which causes the degradation of the transmission signal. And when the transmission signal passes through the High Power Amplifier (HPA), the high Peak-to-Average Power Ratio causes the peaks to enter into saturation region which resulting in the presence of two phenomenon which are in-band radiation (IBR) phenomenon and out-of-band radiation (OBR) phenomenon; the IBR phenomenon will considerably degrade the performance MC-CDMA performance by increasing the bit error rate (BER), and in the OBR side we assist to the presence Adjacent channel interference (ACI). The problem of high PAPR into MC-CDMA during the signal transmission produces many critical system malfunction aspects such as the design complexity of Analog to Digital Converter (ADC) and Digital to Analog Converter (DAC) which play an important role in the transmission interface by converting and realizing reverse function performance during data processing. The high PAPR also affect the power efficiency by increasing the power consumption, increasing the cost, reducing the battery life and increasing the co-channel interference and inter symbol interference (ISI) resulting to the presence of non-linear distortion in high power amplifier (HPA). For this reason, if the PHA is not operated into a linear region by using considerable power back-off it will be logically impossible to keep the out-of-band power below the specified limits, and by following this analysis, the reduction of PAPR leads automatically the reduction of DAC and ADC complexity in the MC-CDMA transmission system. Moreover, the problem of high PAPR in MC-CDMA system requires expensive radio transmitters systems capable to provide high power amplifiers which can operate in a extra large linear range otherwise, the nonlinear signal automatically leads the system to an very high adjacent channel.

Over the last ten years, a variety of PAPR reduction techniques have been proposed to PAPR reduction in MC-CDMA signal [1]; between existing techniques used actually, clipping approach is considered as the more widely adopted approach to realize the PAPR reduction technique; but the main drawback with the use of this technique is that is causes an additional clipping noise resulting in significant out-of-band interference $(\mathrm{OBI})$; this technique is responsible to the clipping and filtering problem which cause the degradation of the performance in MC-CDMA system. The clipping and filtering process consist to combine CDMA and OFDM transmitters as MC-CDMA transmitter system is connected to the clipping \& filtering, and DAC and HPA. The clipping will select the optimum clipping ratio to remove 
the high amplitude peak; but in this processing, the system performance will be considerably affected by reducing the performance and introducing IBR and OBR by filtering. To resolve this problem, the filtering method is adopted to reduce the effect of the IBR and OBR, and the transmitter complexity is increased by adding Fast Fourier Transform (FFT). The purpose of this approach consists to clip the part of the signals that are outside the allowed region.

To fix the issue of additional clipping noise engender with the use of the clipping method, recently a new promising technique called Nonlinear Companding Transform (NCT) was been introduced. And the first introduction of the use of this technique was proposed by X. B. Wang et al (1999) where the main objective consisted to remedy the issue of out-of-band interference (OBI). The scheme developed in this method was based on the use of the traditional $\mu$-law companding approach to perform the traditional clipping [2]. In this evaluation, several methods have been proposed by different authors including SML in [3] which proposed the selective mapping method working with two main advantages such as the use of only one IFFT algorithm at the transmitter side, and the no need of side information for the transmission. The Partial transmit sequence was introduced by P. Varahram et al (2010) [4], which uses a combination of DSI and PTS, this proposal was been implemented in WiMAX application; another technique called active constellation extension were been used in [5] based on pre-scrambling method. The tone reservation (TR) method and tone injection (TI) are having been proposed in [6], [7].

Nevertheless, the common method based on traditional $\mu$ -law Companding technique make the high peak to average (HPA) more sensitive because this Companding technique outperform the clipping. And this new observation opened a new challenge to find an appropriate NCT technique capable to provide proper and sufficient NCT technique. And following this new way, two propositions, the first one appropriated to OFDM scheme based on the statistical distribution, and the second one was especially focused on the optimization technique to perform NCT drawback characteristics. This paper analyses a new idea that is the combination of two recent NCT techniques which are inverse hyperbolic sine function namely $\mathrm{C}^{-1}(),.[8]$, [9] and the clipping concept function which is the revised exponential Companding transform with clipping namely MECCT, [10]: This new method is called HIS-RECT approach.

The remainder of this paper is organized as follows: We present the MC-CDMA PAPR system Analysis in Section II. Section A describes the motivation of PAPR reduction. Section B the related works and system criteria. In the Section $C$ the newly combined Inverse hyperbolic sine function and the revised exponential Companding transform are discussed. In Section D simulation results are presented, and in Section III finally, conclusion are listed.

\section{MC-CDMA PAPR SYSTEM ANALYSIS}

\section{A. The Motivation of PAPR Reduction}

The use of the NCT technique is considered as an attractive and revolutionary way due to its good system performance including its integral simplification in the case of the implementation without restriction of the number of subcarriers, the type constellation, and the presence of the full use of band expansion in the transmission system.

All these characteristics bring NCT to become the selective candidate to PAPR reduction technique.

In MC-CDMA scheme, following the Inverse Fast Fourier Transform (IFFT) at the sender side and Fast Fourier Transform (FFT) at the receiver side, the MC-CDMA system bandwidth passes through several orthogonal subcarriers with narrow bandwidth, and an number of $\mathrm{K}$ users data symbols are modulated by the use of Quadrature Amplitude Modulation (QAM) and transmitted independently on subcarriers where the signal will be converted from parallel to serial $(\mathrm{P} / \mathrm{S})$, and according this formulation, the MC-CDMA signal other the symbol interval can be calculated as follows:

$$
S_{n}=\frac{1}{\sqrt{N}} \sum_{K=0}^{N-1} S K \cdot \exp \left(\frac{j .2 \pi k n}{N}\right), 0 \leq n \leq N-1
$$

where $j=\sqrt{-1}$ is oversampling ratio, and $\mathrm{n}=0,1 \ldots \mathrm{N}-1$ is the time index; In this case, considering $\mathrm{N}$ values which is the number of subcarrier used. $S_{n}$ is is based on central limited theorem and it is a complex Gaussian process with a large value of $\mathrm{N}(\mathrm{e}, \mathrm{g} . \mathrm{N} \geq 64)$. The signal symbol $\mathrm{SN}$ automatically becomes the Gaussian distribution function with Zero mean and a common variance $\sigma^{2}=\mathrm{E}\left\{\left|\mathrm{K}_{\mathrm{X}}\right|^{2}\right\} / 2$, and in this process, the Gaussian Distribution function is write with the probability density as follows:

$$
\left(F \sin (S)=\frac{1}{\sqrt{2 \pi \sigma}} \exp \left(\frac{S^{2}}{2 \sigma}\right)\right.
$$

where $S^{2}$ is the variance of the original of multi-carrier modulation signals, and the constant $s_{n}$ the distribution with cumulative function (CDF); the new calculation of the $S_{n}$ function is taking the following:

$$
F s_{n}(s)=\frac{1}{2}\left(1+\operatorname{er} f\left(\frac{s}{\sqrt{2 \sigma}}\right)\right), \operatorname{erf}(s)=\int_{0}^{x} \frac{2}{\sqrt{\pi}} e^{-y^{2}} d y
$$

We can then define the PAPR of MCM signal $S_{n}$ into a constant symbol period as follows:

$$
\operatorname{PAPR}(s n)=10 \log \frac{\operatorname{Max}\left\{|s n|^{2}\right\}}{\sigma^{2}}(d B)
$$

From equation (3) it is observed that PAPR reduction of MC-CDMA signals is mainly obtained by MC-CDMA signals is mainly obtained by decreasing the maximum instantaneous signal power. For this reason, the new PAPR defined in relation with multi carrier signal is determined as follows:

$$
P A P R=\frac{\max \left|X_{m}\right|^{2}}{\frac{1}{N \operatorname{erf} \int_{0}^{N} \operatorname{erf}|x(d)|^{2} d t}}
$$

By using the companding transform, $\mathrm{S}_{\mathrm{n}}$ are Companded before converted to analog waveforms and amplified by the high power averages. And $S_{C}(n)$ is given by $S_{C}(n)=V\left(S_{n}\right)$, $(\mathrm{n}=0,1, \ldots, \mathrm{N}-1)$, where $\mathrm{C}($.$) is the companding transform$ 
function adopted in the case of MC-CDMA, nevertheless this formulation can also be utilized in OFDM scheme.

We define $\mathrm{X}_{\mathrm{m}}$ as the time samples of an MC-CDMA symbol in the system. and for this affirmation, $m=0$, 1, ....considering that our work is give in the context of downlink, the relation between Crest Factor (CF) and PAPR is given by

$$
C F=\sqrt{P A P R}
$$

And the PAPR of MC-CDMA in downlink context is given by the relation between $L$ users where $N_{C}=\mathrm{L}$ can be calculated as follows:

$$
P A P R \leq 2 \max \frac{\left\{\sum_{k=0}^{k-1}\left|\sum_{l=0}^{l-1} C_{L}^{K} e^{j .2 \pi / T_{S}}\right|\right\}^{2}}{L}
$$

Then, MC-CDMA signals are transmitted into the radio channel. After passing through both AWGN and the frequency selective fading channel.

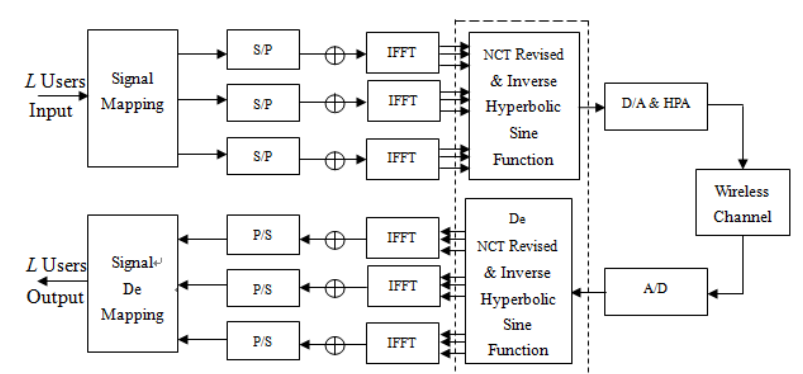

Fig. 1. MC-CDMA system based Revised NCT and the Inverse Hyperbolic Sine Function technique.

Fig. 1 shows the block diagram of MC-CDMA system based on NCT technique which combines the revised NCT and the Inverse Hyperbolic Sine Function.

Where $\mathrm{M}$ is the 1 and $\mathrm{k}$ denote the number of subcarriers used during the parallel transmission process, and by calculation we consider $\mathrm{k}$ with condition $\mathrm{S}_{\mathrm{k}}(0<\mathrm{K}<\mathrm{n}-1)$ as the $\mathrm{K}^{\text {th }}$ the complex modulated symbol in a block of $\mathrm{N}$ information symbols; the Inverse Fast Fourier Transform IFFT which is used to convert the $\mathrm{P} / \mathrm{S}$ is into the frequency interleaving block.

\section{B. Related Woks and Current System Criterias}

\section{1) Related works}

Many works have been proposed other the past decade in the context of the use of Linear Companding Transform (LCT) - PAPR reduction technique [11].

P. Varahram et al. proposed A low complexity partial transmit sequence scheme by use of dummy signals for PAPR reduction technique; this method was based on a combination of DSI and PTS technique which was been also considered as an useful approach for WiMAX application. But the presence of significant number of division and the number of IFFT are still affecting the OFDM signal.

K. D. Chloe et al. proposed a Pre-scrambling method based on a block code which used pre scrambles data sequence for high data speed data transmission; this method performed the use of "low complexity partial transmit sequence scheme" by permit a considerable improvement in the computational complexity.
B. S. Korngold and D. L. Jones used active constellation extension method that dynamically extends outer constellation points in active (data-carrying) channels, within margin-preserving constraints, in order to minimize the peak magnitude. The method offers faster PAR reduction compared to the ACE-POCS approach.

In researches presented above, the reduction of high PAPR effect and the minimize of the OFDM signal are been improved, but the phenomenon such as high BER degradation and out-of-band interference problem still affect the transmission scheme. To resolve this drawback, Yuan Jiand and X. B. Wang introduced a new approach in [12] called New Companding Transform (NCT). The NCT technique is considered as a promise candidate to perform the high PAPR transmission problem present in mobile communication environment. This technique approach was been also improved especially in [13] where the authors proposed a new LCT with more design flexibility than Linear Companding Transform (LCT), and the Non Symmetrical Companding Transform (LNST) was investigated. The authors proposed a LCT technique that has one tone mapping of input and output transformed signal. The proposed scheme degrades Power Spectral Density (PSD), lower PAPR and BER than LNST.

Tao Jiang et al. proposed a new nonlinear companding technique, called "exponential companding", to reduce PAPR of OFDM signals. The exponential companding scheme can offer better PAPR reduction, BER, and phase error performance, and less spectrum side lobes [14], [15]. But its insufficiencies on the performance context was been more improved by B. Sarala et al. whose developed and Modified Exponential Companding Transform with Clipping technique approach to provide more improvement in spectral bandwidth context.

T. Jiang et al. dressed an overview and the state of art for OFDM signal transmission. By the use of clipping and filtering technique;

The use of a novel NCT scheme based on the inverse hyperbolic sine function is proposed and according to this approach, since the power distribution of the Companded signal can be reallocated more reasonably while maintaining an unchanged average power level, significant PAPR reduction as well as an improved bit error rate (BER) performance can be achieved simultaneously. Moreover, by adjusting the variable companding parameters, it also allows more flexibility and freedom in the companding form so that an effective trade-off between the PAPR and BER performance can be offered. Both theoretical analyses and simulations confirm the effectiveness of this novel technique.

\section{2) System criteria}

The criteria of the PAPR reduction are based in an scalable of OFDM PAPR reduction criteria including the new factors to perform HPA; most of them are including: High capability of PAPR reduction, low average power, low implementation complexity, no bandwidth expansion, no BER performance degradation, no need of additional power needed, no spectral spillage, and other factors e.g. effects of the components in the transmitter and their cost considerations.

In this work context, we propose the design criteria of the nonlinear companding transforms, which is based on the 
combination of two recent NCT based techniques which are inverse hyperbolic sine function and the revised exponential companding transform. And analysis of some proposed novel nonlinear companding transforms (HIS-RECT approach). As examples, the proposed novel nonlinear companding transforms can effectively reduce the PAPR of the MCM signals after passing through both AWGN and the frequency selective fading channel, by transforming the power of the original MCM signals into uniform distribution. These novel schemes also have the advantage of maintaining a constant average power level in the nonlinear companding operation. The strict linearity requirements on HPA can then be partially relieved.

\section{Novel NCT Scheme Based Inverse Hyperbolic Technique}

This new idea is a combination of inverse hyperbolic sine function technique and the revised exponential companding transform concept. It generates a new algorithm which as given below:

$$
T_{1}=\frac{\operatorname{median}\left(\left|X_{n}\right|\right)}{\delta_{x_{n}}{ }^{2}}
$$

where $\delta_{\mathrm{x}_{\mathrm{n}}}{ }^{2}$ is the variance of standard deviation atstandard deviation ${ }^{2}$, the modulus symbol of MC-CDMA is given by $\left|\mathrm{X}_{\mathrm{n}}\right|$, with $\mathrm{T}_{1}$ as the threshold value;

Step 2: Calculation of new modulus based combination of HIS-RECT approach can be calculated as follow:

$$
X_{n}^{\prime}=T_{1}+\log \left(\left|X_{n}\right|-T_{1}+1\right)
$$

Step 3: Calculation of Inverse Hyperbolic Syn Function:

$$
x_{m}=x_{n} \text { where } 0 \leq\left|x_{n}\right| \leq T_{1}, x_{n}^{\prime} \text {, when }\left|x_{n}\right| \geq T_{1}
$$

The revised exponential companding transform algorithm is given as follow:

Step 1: Calculation of threshold value (at the receiver side) $\mathrm{T}_{2}$ :

$$
T_{2}=\frac{\operatorname{median}\left(\left|r_{n}\right|\right)}{\sigma_{x_{n}}{ }^{2}}
$$

where $\sigma_{\mathrm{x}_{\mathrm{n}}}{ }^{2}$ is a variance of standard deviation, and $\left|\mathrm{r}_{\mathrm{n}}\right|$ is Modulus of MC-CDMA a received symbol which is the threshold value at the receiver.

Step 2: the New Companding received signal can be described as:

$$
\hat{x}_{n}=r_{n}
$$

\section{Simulation Results}

The evaluation of the performance of MC-CDMA signal is made by using Cumulative Distribution Technique and the Complementary Cumulative Distribution Function (CCDF) is considered as the main constant parameter on measuring the efficiency of our PAPR technique in this paper. The proposed MC-CDMA with the revised exponential companding transform and newly introduced Inverse
Hyperbolic Sine (HIS-RECT) Technique systems are implemented using MATLAB with the following specifications: the number of symbols are 254, 510, 1022 and 4094 symbols; the Inverse Fast Fourier Transform (IFFT) size is 254 , and the number of subcarriers are respectively $126,62,31$; to transmit the signal on the bandwidth we use Pseudo Noise (PN) codes, Reed Solomon code, the modulation process is made with 16-Quadrature Amplitude Modulation (16-QAM) and Amplitude Shift Keying (ASK). The simulations are performed for an MC-CDMA system with PAPR using cumulative distribution of PAPR of MC-CDMA with different codes and companding techniques. And the results are compared with original MC-CDMA with Liner companding, and MC-CDMA with exponential companding, and MCCDMA with newly introduced inverse hyperbolic sine function and the revised exponential companding transform called (HIS-RECT).

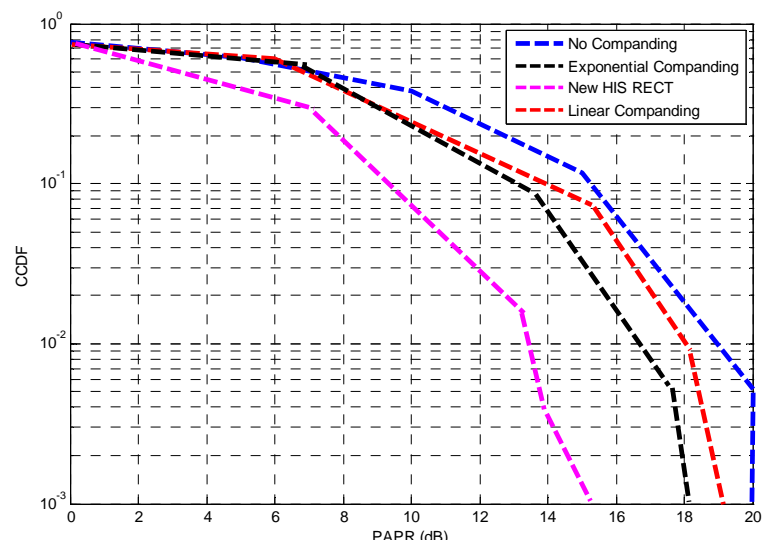

Fig. 2 . Nsym $=510, \mathrm{nfft}=254$, nsub $=62$, ų=0.825, $d=1.1,16 \mathrm{Q} A M$, Reed solomom codes.

The result of MC-CDMA simulation in Fig. 2, Fig. 3, and Fig. 4 show that HIS RECT technique combine to PN/Reed Solomon codes can reduce the PAPR EFFECT by $2 \mathrm{~dB}$, and $2.5 \mathrm{~dB}$ when compared with the original MC-CDMA (traditional companding), and MC-CDMA with exponential companding techniques. And if we increase the number of symbols we also automatically increase the PAPR signal (up to $2.5 \mathrm{~dB}$ ).

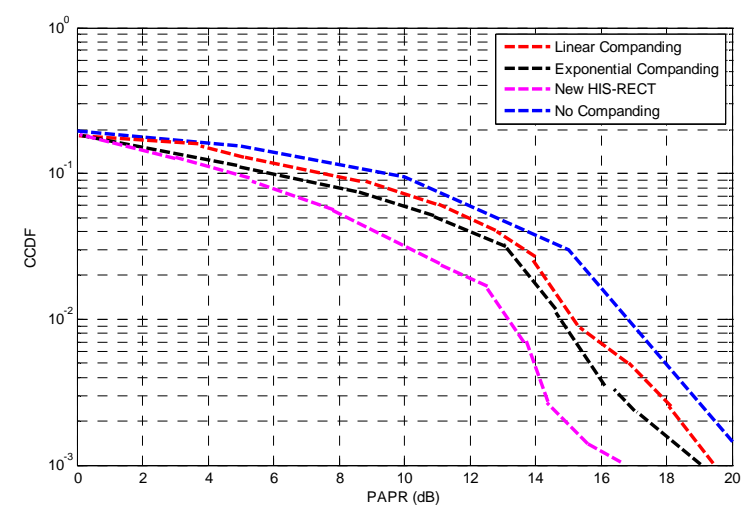

Fig. 3. Nsym $=510, n f f t=254, \mathrm{nsub}=126, \mathrm{u}=0.825, \mathrm{~d}=1.1,16 \mathrm{QAM}$, Reed solomom codes.

The Fig. 5, 6 and 7 show that, using MC-CDMA with PN code and HIS-RECT technique, PAPR is reduced by $2.5 \mathrm{~dB}$, and $2.0 \mathrm{~dB}$ by comparing with the traditional Non Companding technique used in the same context. 


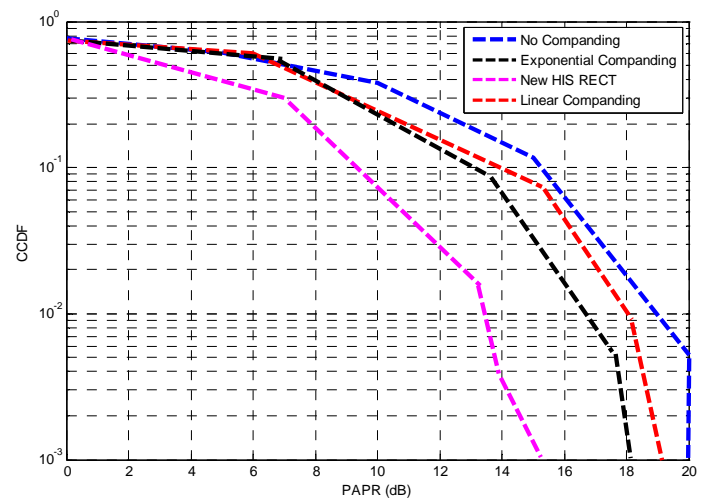

Fig. 4. Nsym=1022, $\mathrm{nfft}=254, \mathrm{nsub}=126, \mathrm{u}=0.825, \mathrm{~d}=1.1,16 \mathrm{QAM}$, Reed solomom codes.

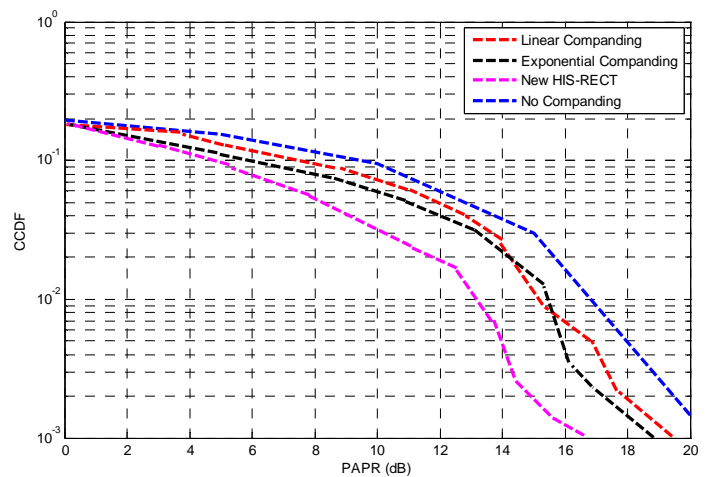

Fig. 5. Nsym $=510, n f f t=254, n s u b=62, \mathrm{u}=0.825, d=1.1,16 Q A M, P N$ codes

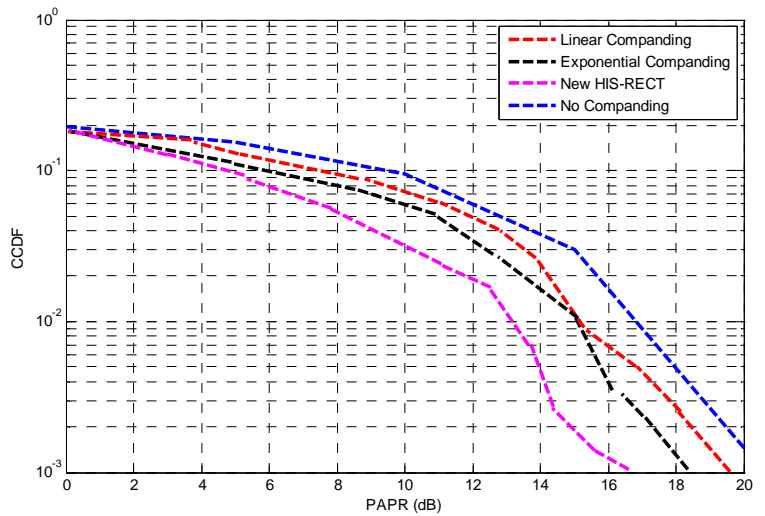

Fig. 6. Nsym=510, nfft $=254, \mathrm{nsub}=126, \mathrm{u}=0.825, \mathrm{~d}=1.1,16 \mathrm{QAM}, \mathrm{NC}$ codes.

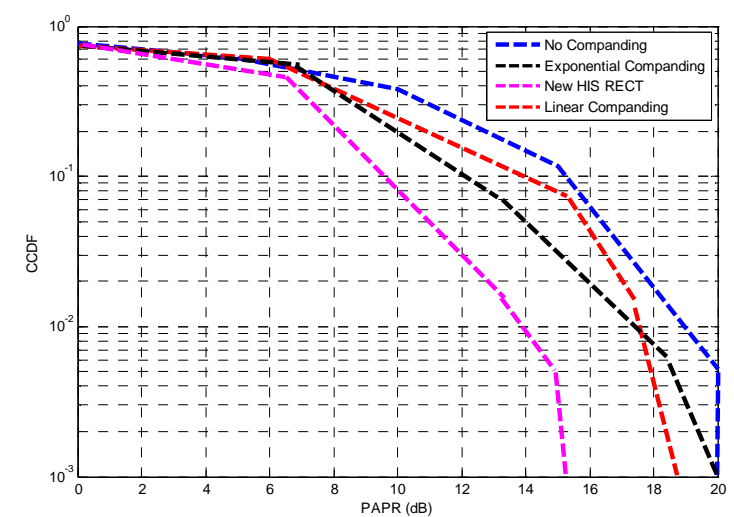

Fig. 7. $\mathrm{Nsym}=4094, \mathrm{nfft}=254, \mathrm{nsub}=126, \mathrm{u}=0.825, \mathrm{~d}=1.1,16 \mathrm{QAM}, \mathrm{NC}$ codes.

The use of Reed Solomon Codes combined to MC-CDMA with HIS-RECT technique show in Fig. 8, 9, 10 and 11 that the PAPR phenomenon can be reduced by $1.5 \mathrm{~dB}$ and 1.75
$\mathrm{dB}$ when compared with traditional MC-CDMA companding techniques, the no companding technique and the MC-CDMA with Exponential Companding. And if the number of symbols is increased, the PAPR is further reduced by $0.5 \mathrm{~dB}$. If we double the number of subcarriers we got a PAPR which is increased by $2.5 \mathrm{~dB}$.

The simulation results of Power Spectral Density shows in Fig. 12. that the HIS-RECT based MC-CDMA technique has $11 \mathrm{~dB}$ less in lower side and main lobe when compared with the traditional MC-CDMA technique, linear technique and exponential technique. The MC-CDMA with linear system has less mean amplitude and the system maintains constant the main lobe bandwidth compared to other MC-CDMA systems implemented in the same conditions.

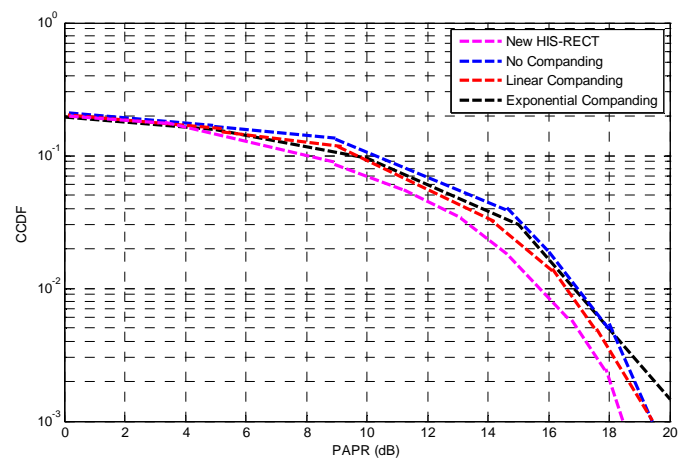

Fig. 8 . Nsym=254, nfft= 254, nsub=62, $\mathrm{u}=0.825, \mathrm{~d}=1.1,16 \mathrm{QAM}, \mathrm{NC}$ codes.

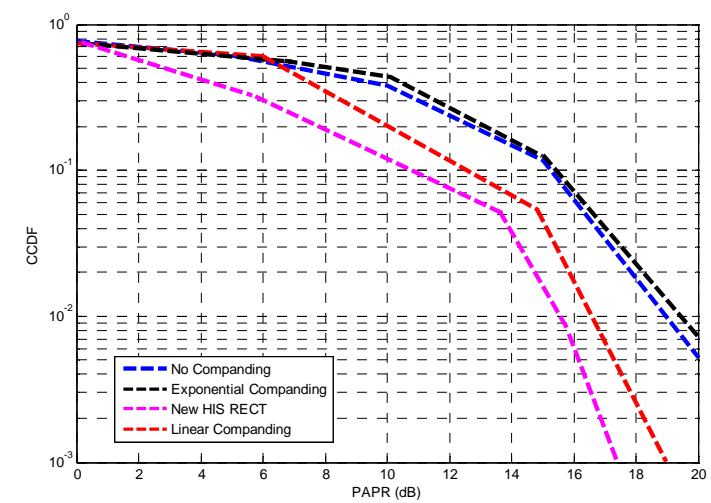

Fig. 9. Nsym=254, $n f f t=254, n s u b=30, u=0.825, d=1.1,16 Q A M$, reed solomon codes.

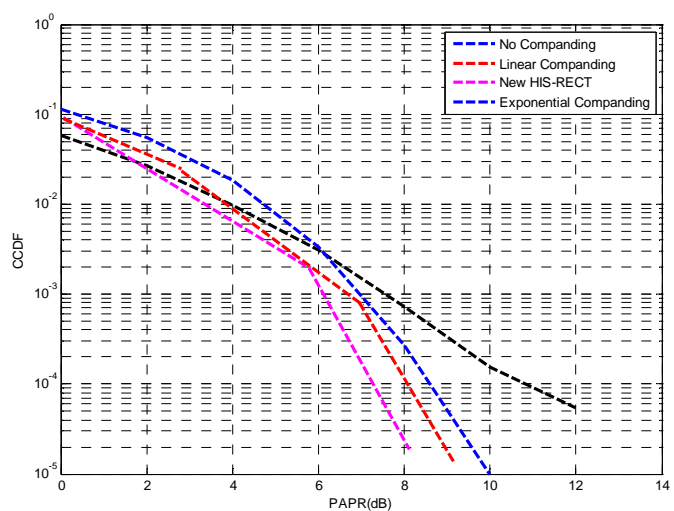

Fig. 10. Nsym=4094, $n f f t=254, n s u b=30, u=0.825, d=1.1,16 Q A M$, reed solomon codes.

The result of BER simulations by using Reed Solomon / PN codes and HIS-RECT combined to MC-CDMA system with AWGN channel is shown in the Fig. 12. Found BER 9.2 at $10.1 \mathrm{~dB}$, the MC-CDMA with linear companding found 
BER is $0.8^{*} 9.2$ at $10.1 \mathrm{Db}$. And a newly introduced HIS-RECT with MC-CDMA technique obtains better BER when compared with the traditional combined MC-CDMA with linear companding technique.

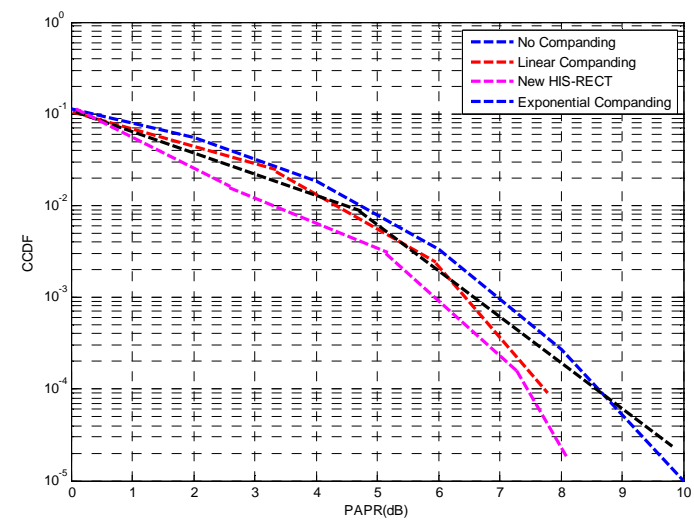

Fig. 11. Nsym=4094, nfft $=254, \mathrm{nsub}=62, \mathrm{u}=0.825, \mathrm{~d}=1.1,16 \mathrm{QAM}$, reed solomon codes.

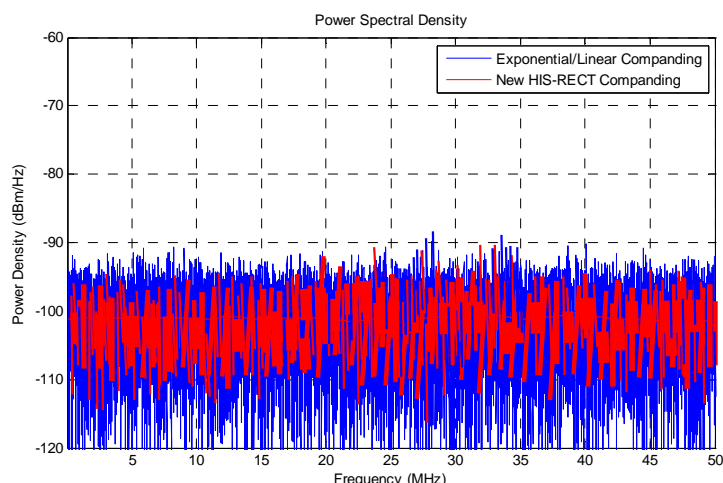

Fig. 12. Power spectral density comparison.

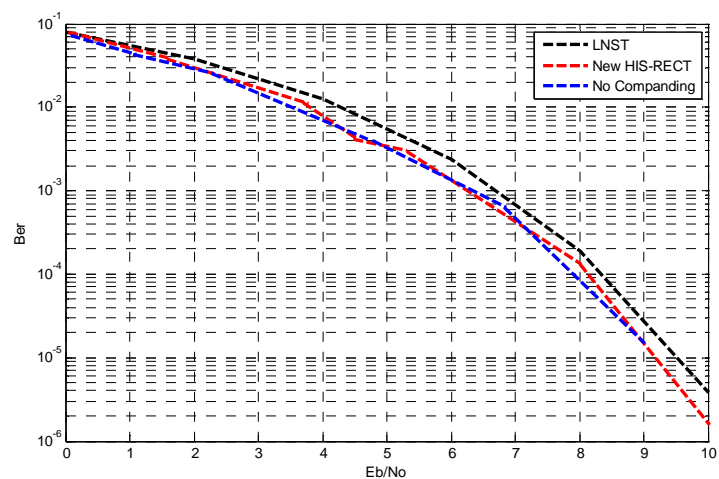

Fig. 13. BER analysis of new introduced HIS-RECT with linear companding technique.

\section{CONCLUSIONS AND FUTURE WORK}

Both the PAPR reduction and moderate BER performance degradation are critical challenges for a well-designed NCT method in MC-CDMA MIMO system. In this paper, a novel MC-CDMA system used HIS RECT is introduced to reduce PAPR about $2.5 \mathrm{~dB}$ for Reed Solomon and codes and at 1.5 $\mathrm{dB}$ for $\mathrm{NC}$ codes, decrease the BER over linear companding technique and improve the spectrum efficiency. The new approach found that the HIS-RECT based MC-CDMA has $11 \mathrm{~dB}$ less in side and main lobe when compared with the MC-CDMA based traditional linear companding. The BER performance is improved and PAPR is also reduced. This novel technique is very efficient, easily implemented and does not require the use of any sophistic program.

The future work will be focused on the low data rate optimization and less complexity efficient PAPR reduction of MC-CDMA system. This work is implemented by used Raleigh Fading Channel.

\section{ACKNOWLEDGMENT}

We would like to present our thanks to China Scholarship Council (CSC No: 2013266T12) port our study financially and Wuhan University of Technology which make the best conditions for our studying. The authors greatly appreciate the anonymous reviewers for carefully reading this paper and suggesting many helpful comments on improving the original manuscript.

\section{REFERENCES}

[1] B. Sarala, D. S. Venkateswawarulu, and B. N. Bhandari, "Overview of MC-CDMA PAPR reduction techniques," International Journal of Distributed and Parallel Systems (IJDPS), vol. 3, no. 2 , pp. 193-206, March 2012.

[2] R. Manjith, S. C. Ramesh, and M. Mohamed, "PAPR reduction in OFDM and MC-CDMA system using nonlinear companding Techniques," in Proc. International Conference on Electrical and Electronical, pp. 1-5, July 2010.

[3] Y. Jie et al., "A modified selected mapping technique to reduce the peak-to-average power ratio of OFDM signal," in Proc. International Conference on Consumer Electronics, IEEE Trans. Consumer Electron, vol. 53, no. 3, pp. 846-851, Aug. 2007.

[4] K. D. Choe, S. C. Kim, and S. K. Park, "Pre-scrambling method for PAPPAPR reduction in OFDM communication systems," IEEE Trans. Consumer Electron., vol. 50, no. 4, pp. 1044-1048, Nov. 2014.

[5] K. D. Choe, S. C. Kim, and S. K. Park, "Pre-scrambling method for PAPR reduction in OFDM communication systems," IEEE Trans. Consumer Electron., vol. 50, no. 4, pp. 1044-1048, Nov. 2014.

[6] J. Tellado, "Peak to average power ratio reduction for multicarrier modulation," PhD thesis, University of Stanford, Stanford, 1999.

[7] S. S. Yoo, S. Yoon, S. Y. Kim, and I. Song, "A novel PAPR reduction scheme for OFDM systems: Selective mapping of partial tones (SMOPT)," IEEE Trans. Consumer Electronics, vol. 52, no. 1, pp. 40-43, Feb. 2006.

[8] Y, Wang, L. H. Wang, and B. Ai, "Nonlinear companding transform technique for reducing PAPR of OFDM Signal," International Conference on Transaction on Consumers Electronics, vol. 58, no. 3, pp. 1-5, August 2012.

[9] T. Jiang and Y. Yang, "Exponential companding technique for PAPR reduction in OFDM systems," presented at IEEE International Conference on Transactions on Broadcasting, June 2005.

[10] B. Sarala, D. S. Venkateswarulu, and B. N. Bhandari, "MC-CDMA PAPR reduction using a modified exponential companding transform with clipping," Global Journal of Researches in EngineeringElectrical and Electronics Engineering, volume 13, issue 10, pp. 1-7, 2013.

[11] S. A. E. Mohamed, "Linear companding transform for the reduction of peak-to-average power ratio of OFDM signals," IEEE Transactions Broadcast, vol. 55, no. 1, pp. 155-160, 2009.

[12] X. B. Wang, T. T. Tjhung, and C. S. Ng, "Reduction of peak-to-average power ratio of OFDM system using a companding technique," IEEE Trans. Broadcast, vol. 45, no. 3, pp. 303-307, Sep. 2009.

[13] Y. Jiang, "New companding transform for PAPR Reduction in OFDM," IEEE Commun. Lett., vol. 14, no. 4, pp. 282-284, Apr. 2011.

[14] T. Jiang, Y. Yang, and Y. Song, "Exponential companding technique for PAPR reduction in OFDM signals," IEEE Transactions Broadcast, vol. 51, no. 2, pp. 244-248, 2005.

[15] T. Jiang and Y. Wu, "An overview: Peak-to-average power ratio reduction techniques for OFDM signals," IEEE Trans. Broadcast, vol. 54, no. 2, pp. 257-268, Jun. 2010. 


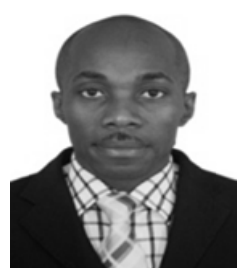

Mbembo Loundou Varus was born in Gabon in 1981. He received his BS degree in economic sciences in National University of Omar Bongo Ondimba in 2006, and the MS degree In information engineering in 2012 in Beijing University of Technology-China. He is currently $\mathrm{Ph} . \mathrm{D}$ student in Wuhan University of Tchenology, China. His Research area including principles and technologies of modern communication network, on mobile high data rates, MIMO/SIMO antenna systems and antenna multiplexing transmission design.

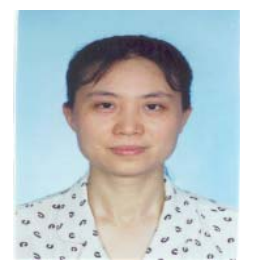

Jie Yang was born in Wuhan, China, in 1960. She received her BS degree in communication engineering from Xidian University, China, in 1982 and the MS degree in computer and automation from Wuhan Transportation University, China, in 1988 She received the Ph.D. degree in electronic engineering from the Shanghai Jiao Tong University, China, in 1999. She has or of School of Electronics and Information at Wuhan University of Technology since 1999. Her current research interests include image processing, information hiding, cryptography and multimedia communication.

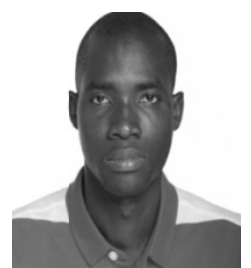

Nouhoum Abdoul Galeb was born in Djougou $(\mathrm{BENIN})$. He received an engineer degree in telecommunications systems from Universite 08 Mai 45 of Guelma (ALGERIA) in 2008. From 2008 to 2012 he has worked for ministry of communication and information technology in Benin. Since September 2014 he is Ph.D. candidate at School of Information Engineering Wuhan University of Technology.

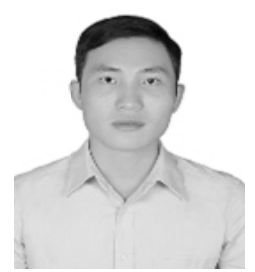

Dong Doan Van received the B.S. degree in electronic and telecommunication from Ho Chi Minh City University of Transport, Viet Nam in 2010. He had graduated the M.S. degree in electronic and information system from Huazhong University of Science and Technology (HUST), Wuhan, China in 2014, he is currently a Ph.D. Student at Wuhan University of Technology, Wuhan-Hubei, China. His research includes content centric network, wireless sensor network, signal processing and telecommunication. 